\title{
The Impact of Different Thyroid Surgery Routes on the Function of Parathyroid G Zhu, X Zou*
}

\begin{abstract}
Objective: To explore the impact of three different surgery routes for thyroid cancer on the function of parathyroid.

Methods: Three groups were included: group A-gland lobe removal of one side + ipsilateral lymph nodes dissection at the central neck area $(n=62)$; group B-total thyroid removal + unilateral lymph nodes dissection at neck IV area with/without ipsilateral dissection of neck II-V area $(n=193)$; group C-total thyroid removal + bilateral neck VI dissections with/without lymph nodes dissection of ipsilateral neck IIV area $(n=60)$. Serum parathyroid hormone $(\mathrm{PTH})$ and calcium were tested before and after surgery.

Results: For all groups, both PTH and calcium levels dropped after surgery. There is no difference for serum PTH and calcium one or four days after surgery between group B and C. Group A had much lower incidence of hypocalcaemia or hypoparathyroidism, compared B or C $(\mathrm{p}<0.05)$. In group A, artery and recurrent vein of parathyroid were spared during the surgery, suggesting the importance of this regard.

Conclusion: The larger of surgical area, the higher incidence of hypoparathyroidism. The incidence of hypoparathyroidism can dramatically drop if artery and recurrent vein of parathyroid are spared during surgery, as indicated in gland lobe removal with ipsilateral lymph nodes dissection.
\end{abstract}

Keywords: Hypocalcaemia, parathyroid function, thyroid surgery routes

From: Department of General Surgery, Jiang Yuan Hospital Affiliated with the Jiangsu Institution of Nuclear Medicine (Key Laboratory of Nuclear Medicine, Ministry of Health, Jiangsu Key Laboratory of Molecular Nuclear Medicine), 20 Qianrong Rd. Wuxi, 214063, Jiangsu, China

Correspondence: Dr X Zou, Department of General Surgery, Jiang Yuan Hospital Affiliated with the Jiangsu Institution of Nuclear Medicine, 20 Qianrong Rd. Wuxi, 214063, Jiangsu, China. Tel: +86-0510-85514482-5700, Fax: None. E-mail: zouxian11new@ sina.com 


\section{Thyroid Surgery Routes on PTH}

\section{INTRODUCTION}

The incidence of thyroid papillary carcinoma increased significantly in the past years (1). It is very easy for this type of cancer metastasize to the lymph nodes of neck IV area (2). Once it is relapsed at this area, the second surgery is generally required, which is usually complicated with high incidence of recurrent pharyngeal nerve and parathyroid (3). Thus, more and more surgeon accepted the surgery of thyroid removal with ipsilateral lymph nodes dissection at the first place.

Although a growing studies have been focussed on the impacts of different surgical routes of the thyroid on the function of parathyroid, it remains unclear which route is most helpful for retaining the function of the parathyroid and how.

One major route is the partial thyroid removal with limited ipsilateral lymph nodes dissection (4). In this surgery, as a small surgical field is involved with a rather limited removal and dissection, the vessels of the parathyroid can be spared as much as possible. The key vessel is the recurrent vein of parathyroid, which can be spared with cautious surgery (5). Yet, little is known if this is associated with better parathyroid function.

In this study, we aimed at exploring the different impacts of different surgical routes for thyroid papillary carcinoma on the function of parathyroid, and how to protect the parathyroid during the surgery for thyroid removal and lymph nodes dissection.

\section{METHODS AND MATERIALS}

General information and inclusion

Among those admitted to the Department of general surgery at Jiangyuan Hospital affiliated to medical atom research institute of Jiangsu province during January of 2011 to December 
of 2012, a total of 315 patients with thyroid papillary carcinoma were enrolled into this retrospective study. There were 72 males and 242 females (23\% and 77\%, respectively), aged at $17-69$ years old with a mean age of 44 years.

Inclusion: those without neck surgery or kidney disease; those having thyroid lobe removal with neck IV area lymph nodes dissection due to thyroid papillary carcinoma; those with normal serum calcium and parathyroid hormone levels before surgery without calcium supplements; asymptomatic of numbness or convulsion of the limbs or face. Ultrasound and fine needle aspiration were performed in all subjects and pathology was performed in some subjects. Routine frozen pathology was performed during surgery.

Groups and surgical routes

Surgical areas were determined according to the following criteria.

Group A ( $\mathrm{n}=62)$-thyroid lobe removal: 15 years $\leq$ age $\leq 45$ years; diameter of the carcinoma $\leq 1.0 \mathrm{~cm}$, without invasion to the capsule; no family history of thyroid cancer or neck radiation; no metastasis to neck lymph nodes per imaging examination before the surgery and no significant swollen lymph nodes revealed during surgery.

Group B and C ( $\mathrm{n}=193,60$, respectively)-total thyroid removal with neck IV area lymph nodes dissection (group B) or neck II-V area lymph nodes dissection (group C): age is less 15 years or over 45 years; diameter of the carcinoma is over $1.0 \mathrm{~cm}$, with history of neck radiation, or family history of thyroid cancer, bilateral thyroid nodules, local metastasis to lymph nodes or remote metastasis, invasive pathology and papillary carcinoma at the isthmus of the thyroid. Neck IV area lymph nodes dissection will be performed in all subjects (group B and C). 


\section{Thyroid Surgery Routes on PTH}

Those with isthmus metastasis will be dissected bilaterally. Those with lymph nodes metastasis on imaging before surgery or needle pathology will be dissected for II-V areas.

\section{Measurements}

One day before surgery, one and four days after surgery, serum calcium and PTH will be determined. The symptoms such as numbness or convulsion of the limbs or face will be evaluated as well.

Parathyroid hormone was determined with E70 electrochemical luminescence immunity auto-analyser (Roche Inc), with a normal range of 15-65 ng/L. Calcium level < $15 \mathrm{ng} / \mathrm{L}$ was defined as hypoparathyroidism.

Calcium was tested with P800 automatic biochemical analyser (Roche Inc), with a normal range of 2.0-2.8 mmol/L. PTH level $<2.0 \mathrm{mmol} / \mathrm{L}$ was determined as hypocalcaemia.

Statistical analysis

SPSS 17.0 was used for data analysis. Comparison was performed before, one and four days after surgery for serum calcium and PTH among three groups with Analysis of variance (ANOVA). Incidence of hypocalcaemia and hypoparathyroidism was analysed with $X^{2}$ test. Data was presented as mean \pm SE. $p<0.05$ was regarded as statistical significant.

\section{RESULTS}

Changes of serum calcium and PTH before, one and four days after surgery in all groups. All the subjects had normal levels of PTH and calcium before surgery, and none of them required for prophylactic calcium supplements after surgery. When symptomatic for hypocalcaemia, 
the subjects were given oral or infusion of calcium. All the subjects had normal levels of PTH and calcium six months after the surgery and none had permanent hypoparathyroidism.

As shown in Figure. 1A, serum PTH was significantly decreased after surgery in all groups $(p<0.05)$, and there is no difference between one and four days after surgery.

Figure 1A

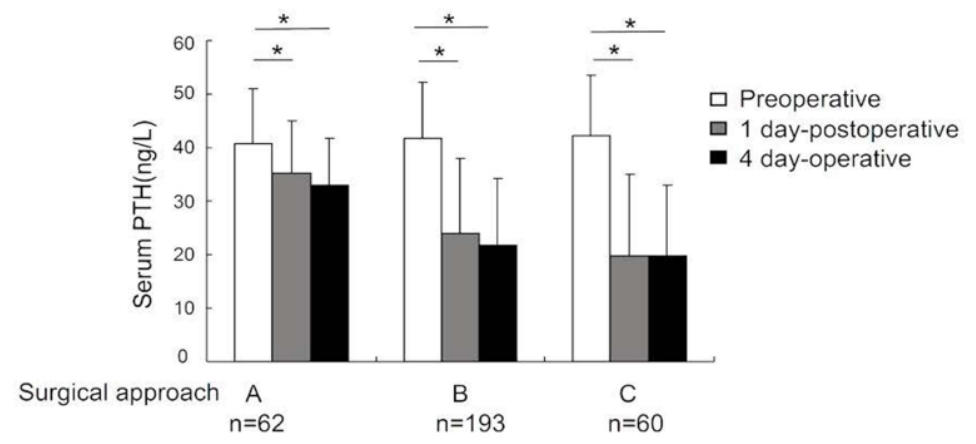

Fig. 1A: the changes of serum level of calcium after different thyroid surgical routes (mean $\pm \mathrm{SE}$ ).

${ }^{*} \mathrm{p}<0.05$, compared with preoperative level.

Figure. 1B showed serum calcium levels, which are consistent with those of PTH. This suggested that the surgery of thyroid may injure parathyroid and have a negative impact on its function.

Figure 1B

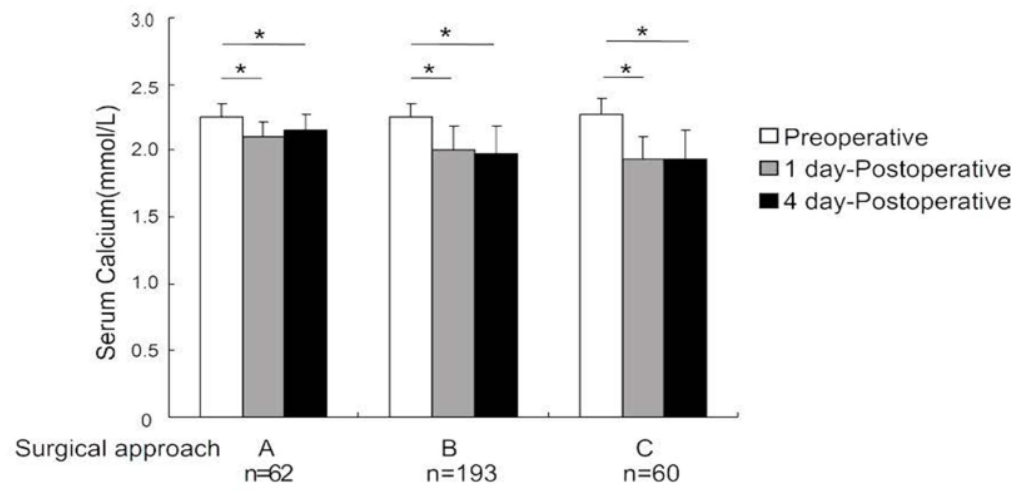

Fig. 1B: $* p<0.05$, compared with preoperative level. 


\section{Thyroid Surgery Routes on PTH}

Comparison of changes PTH and calcium before surgery and four days after surgery among groups.

As shown in Table 1, the serum calcium level dropped dramatically in all groups after surgery, most significant in group C, followed by group B and the least in group A $(p<0.05$ for C vs B, C vs A). For PTH changes, there was no difference for groups B and C. Group A had significant less changes than group B or $\mathrm{C}(p<0.05)$, suggesting thyroid lobe removal + ipsilateral lymph nodes dissection had less impact in deteriorating function of parathyroid.

Table 1. Serum PTH and calcium of preoperative and four-day postoperative in different thyroid surgical routes. (Mean $\pm \mathrm{SE}$ )

\begin{tabular}{lccc}
\hline surgical routes & $\mathbf{N}$ & $\begin{array}{c}\text { Serum PTH } \\
(\mathbf{n g} / \mathbf{L})\end{array}$ & $\begin{array}{c}\text { Serum } \\
\text { Calcium (mmol/L) }\end{array}$ \\
\hline A & 62 & $7.73 \pm 1.73$ & $0.14 \pm 0.02$ \\
B & 193 & $19.90 \pm 1.27$ & $0.28 \pm 0.02$ \\
C & 60 & $22.67 \pm 2.42$ & $0.34 \pm 0.03$ \\
\hline
\end{tabular}

Note: $p<0.05$ among groups for calcium level, highest in group $\mathrm{C}$ and lowest in group $\mathrm{A}$. There is no difference between B and C for PTH. PTH is lower in group A than those of B or C $(p<0.05)$.

Comparison of incidence of hypocalcaemia, hypoparathyroidism and symptomatic hypocalcaemia among groups. Diagnosis was determined as mentioned in the methods. As shown in Table 2, among all subjects, 106 had hypoparathyroidism, with an incidence of $33.65 \%$; 170 had hypocalcaemia, with an incidence of $53.97 \%$ and 108 had symptomatic hypocalcaemia, with an incidence of $34.29 \%$. There was no difference between group B and C 
for the above mentioned incidences. But the incidences in group A were much lower than those of group B or C $(p<0.05)$.

Table. 2: The incidences of hypoparathyroidism, hypocalcaemia and symptomatic hypocalcaemia

\begin{tabular}{ccccc}
\hline surgical routes & N & Low serum PTH & Hypocalcaemia & $\begin{array}{c}\text { Symptomatic } \\
\text { hypocalcaemia }\end{array}$ \\
\hline A & 62 & 0 & $11(17.74 \%)$ & 0 \\
B & 193 & $77(39.9 \%)$ & $118(61.14 \%)$ & $78(40.41 \%)$ \\
C & 60 & $29(48.33 \%)$ & $41(68.33 \%)$ & $30(50 \%)$ \\
\hline
\end{tabular}

Note: For the incidences of hypoparathyroidism, hypocalcaemia and symptomatic hypocalcaemia, there was no difference between group B and C. Group had much lower incidence compared B or C $(p<0.05)$.

\section{DISCUSSION}

Hypoparathyroidism is a common complication of thyroid surgery, affecting life quality of postoperative patients badly (6). During the surgery, the damage and injury of parathyroid vessels is a major reason for postoperative hypoparathyroidism $(7,8)$. Our study showed that different calcium and PTH level with different surgical routes for thyroid, suggesting the importance of the routes. It demonstrated that in group B and C, which had a rather large surgical field, the incidences of hypoparathyroid, calcaemia and symptomatic calcaemia were higher. In group C, with a partial thyroid gland removal, the above mentioned incidences were much lower. The major difference between these routes are that in group $\mathrm{C}$, there was better protection for more than two pieces of parathyroid glands, especially the protection of recurrent vein of thyroid. This is consistent with the previous studies by Thomusch et al $(9,10)$. This protection is more 


\section{Thyroid Surgery Routes on PTH}

effective and important than those with autologous graft, as suggested by the report by Kihara et al $(11,12)$. Both our and other studies suggested the importance of maintaining original parathyroid glands as well as their blood and nerve supplies, with a partial removal of the thyroid gland and limited dissection of lymph nodes (13-18).

The most important vessel and nerve of parathyroid are the recurrent vein and nerve, which are located behind the thyroid gland and thus easily injured during the procedure $(20,21)$. As we hypothesized, with limited surgical field and removal, these vessel and nerve can be spared as much as possible. Our result supported this hypothesis, as shown in group A, in which the partial removal has spared both the vessel and nerve significantly. Thus, it has a great potential to retain the parathyroid function and benefit the patients from the long run. This is consistent with some previous reports $(22,23)$.

Yet, our study had limitations. In our study, it did show inconsistence of changes of PTH or calcium. As serum calcium level may be affected by multiple factors, such as fasting, inadequate fluids, drugs, squeezing during surgery, surgical stress, and hormones changes, we believe that serum PTH may be a better indicator of parathyroid function.

In conclusion, our study suggested that partial removal of thyroid with limited dissection of the lymph nodes is more effective route to avoid postoperative hypoparathyroid, largely due to the protection of recurrent vein and nerve of the gland.

\section{ACKNOWLEDGMENT}

None.

Conflict of Interest: The authors declared no conflict of interest. 


\section{REFERENCES}

1. Zou X, Zhu GH, Hu BS. Risk factors analysis for level VI lymoph node metastasis in papillary thyroid (J). China Oncology 2012; 22: 770-3.

2. Lee YS, Kim SW, Kim SW, Kim SK, Kang HS, Lee ES, et al. Extent of routine central lymph node dissection with small papillary thyroid carcinoma. World J Surg 2007; 31: 1954-9.

3. Sywak M, Cornford L, Roach P, Stalberg P, Sidhu S, Delbridge L. Routine ipsilateral level VI lymphadenectomy reduces postoperative thyroglobulin levels in papillary thyroid cancer. Surgery 2006; 140: 1000-7.

4. Guidelines taskforce on thyroid nodules and differentiated thyroid cancer. Chin J Endocrinol Metab 2012; 28: 779-97. DOI: 10.3760/cma. j. issn. 1000-6699.2012. 10. 002.

5. Cavicchi O, Piccin O, Caliceti U, De Cataldis A, Pasquali R, Ceroni AR. Transient hypoparathyroidism following thyroidectomy: a prospective study and multivariate analysis of 604 consecutive patients(J). Otolaryngology-Head and Neck Surgery 2007; 137: $654-8$.

6. Nahas ZS, Farrag TY, Lin FR, Belin RM, Tufano RP. A safe and cost-effective short hospital stay protocol to iden-tify patients at low risk for the development of significant hypocalcemia after total thyroidectomy. Laryngoscope 2006; 116: 906-10.

7. Lindblom P, Westerdahl J, Bergenfelz A. Low parathyroid hormone levels after thyroid surgery: a feasible predictor of hypocalcemia. Surgery 2002; 131: 515-20. 


\section{Thyroid Surgery Routes on PTH}

8. Paek S H, Lee YM, Min SY, Kim SW, Chung KW, Youn YK. Risk factors of hypoparathyroidism following total thyroidectomy for thyroid cancer (J). World journal of surgery 2013; 37: 94-101.

9. Tolone S, Roberto R, del Genio G, Brusciano L, Parmeggiani D, Amoroso V, et al. The impact of age and oral calcium and vitamin D supplements on postoperative hypocalcemia after total thyroidectomy. A prospective study (J). BMC Surgery 2013; 13: S11.

10. Pesce CE, Shiue Z, Tsai HL, Umbricht CB, Tufano RP, Dackiw AP, et al. Postoperative hypocalcemia after thyroidectomy for Graves' disease . Thyroid 2010; 20: 1279-83.

11. Duclos A, Peix JL, Colin C, Kraimps JL, Menegaux F, Pattou F, et al. Influence of experience on performance of individual surgeons in thyroid surgery: prospective cross sectional multicentre study (J). BMJ: British Medical Journal 2012; 10: 344.

12. Thomusch O, Machens A, Sekulla C, Ukkat J, Brauckhoff M, Dralle H. The impact of surgical technique on postoperative hypoparathyroidism in bilateral thyroid surgery: a multivariate analysis of 5846 consecutive patients. Surgery 2003; 133: 180-5.

13. Page C, Strunski V. Parathyroid risk in total thyroidectomy for bilateral, benign, multinodular goiter: report of 351 surgical cases. J Laryngol Otol 2007; 121:237-41.

14. Michael A, Rosenbaum, Christopher R. Central Neck Dissection for Papillary Thyroid Cancer (J). Arch Otolaryngol Head Neck Surg 2009; 135: 1092-7.

15. Yong Sang Lee, Kee-Hyun Nam, Hang-seok, Chang HS, Park CS. Postoperative Complications of Thyroid Cancer in a Single Center Experience (J). J Korean Med Sci 2010; 25: 541-5. 
16. Bilezikian JP, Khan A, Potts Jr JT, Brandi ML, Clarke BL, Shoback D, et al. Hypoparathyroidism in the adult: epidemiology, diagnosis, pathophysiology, targetorgan involvement, treatment, and challenges for future research. J Bone Miner Res 2011; 26: 2317-37.

17. Lodovico R, Nicola A, Paolo B, De Palma M, Gulino G, Nasi PG, et al. Complications of Thyroid Surgery: Analysis of a Multicentric Study on 14,934 Patients Operated on in Italy over 5 Years. (J). World J Surg 2004; 28: 271-6.

18. McHenry CR, Speroff T, Wentworth D, Murphy T. Risk factors for posthyroidectomy hypocalcemia. Surgery 1994; 116: 641-7.

19. Kihara M, Yokomise H, Miyauchi A, Matsusaka K. Recovery of parathyroid function after total thyroidectomy(J). Surg Today 2000; 30: 333-8.

20. Oertli D, Udelsman R. Surgery of the Thyroid and Parathyroid Glands (M). New York: Springer-Verlag 2007; 85-6.

21. Roh JL, Park JY, Park CI. Prevention of postoperative hypocalcemia with routine oral calcium and vitamin D supplements in patients with differentiated papillary thyroid carcinoma undergoing total thyroidectomy plus central neck dissection (J). Cancer 2009; 115: $251-8$.

22. Tolone S, Roberto R, Genio Gd, Pedroni C, Renna L, Gradoni P. Complications of Central Neck Dissection in Patients with Papillary Thyroid Carcinoma:Results of a Study on 1087 Patients and Review of the Literature. BMC Surgery 2013; 13: S11.

23. Huang SM. Do we overtreat post-thyroidectmy hypocalcemia? (J). World J Surg 2012; 36: $1503-8$. 